\title{
Prototype of FDD/TDD Dual Mode LTE Base Station and Terminal Adaptor utilizing TV White-spaces
}

\author{
Takeshi Matsumura ${ }^{1}$, Kazuo Ibuka $^{1}$, Kentaro Ishizu ${ }^{1}$, Homare Murakami $^{1}$ and Hiroshi Harada ${ }^{2,3}$ \\ ${ }^{1}$ Smart Wireless Laboratory, Wireless Network Research Institute, NICT, Yokosuka, Japan \\ ${ }^{2}$ Social ICT Research Center, NICT, Koganei, Japan \\ ${ }^{3}$ Dept. of Communications and Computer Engineering, Graduate School of Informatics, Kyoto University, Kyoto, Japan \\ matsumura@nict.go.jp
}

\begin{abstract}
Recently, a spectrum resource shortage problem has been aggravated in a cellular system due to growth of service or application business based on high-speed and high-capacity communication. To solve this problem, data traffic offloading to "white-spaces" has been promoted worldwide. In particular, white-spaces in the TV band (TVWS, TV White-spaces) has attracted attentions for securing more channels to existing communication systems and rules for a secondary use of the TVWS have been established in the USA, the UK, Japan, etc. NICT has researched and developed TVWS communication systems that consist of a white-space database, a network manager and some prototyped TV band devices based on WLAN systems including the IEEE802.11af draft standard, however, TV band devices based on current cellular systems are also necessary for further investigation of cellular traffic offloading to the TVWS. In this study, TDD/FDD dual mode LTE base station and terminal adaptor utilizing TVWS are prototyped by applying the UHF converter technology and RF performance is characterized.
\end{abstract}

Keywords-component; TV white-spaces; spectrum sharing; TVWS LTE; prototype; FDD; TDD

\section{INTRODUCTION}

Recently, mobile terminals represented by smart phones and tablet computers have become widely used and a spectrum resource shortage problem has been aggravated due to an increase in data traffics based on high-speed and high-capacity communication for a video hosting service, a cloud computing service, etc. To solve this problem, traffic offloading to other communication systems such as a WLAN (Wireless Local Area Network) system is one of effective options, however, communication systems enabling high-speed communication are also critically crowded. In such a situation, white-spaces, which are allocated to existing communication systems but temporally or spatially unused, are expected to secure alternative channels to crowded mobile communication systems. In particular, communication systems utilizing TVWS have been researched and developed worldwide and some international standardization activities have been actively

This research was conducted under contracts of R\&D on "dynamic spectrum access in multiple frequency bands for efficient radio resource utilization" and "network construction using car-to-car communications technologies" with the Ministry of Internal Affairs and Communications, Japan. promoted; e.g. IEEE802.11af for WLAN system [1] and IEEE802.22 for WRAN (Wireless Regional Area Network) [2]. Meanwhile, protection of TV broadcasting (primary users) is an important issue, and rules for a secondary use of the TVWS have been established by the FCC in the USA [3], the Ofcom in the UK [4], etc. In Japan, a guideline of area broadcasting systems and wireless microphones utilizing the TVWS has been also released [5].

There has been a growing expectation in exploitation of the TVWS by cellular communication systems, and use cases and scenarios related to the secondary use of the TVWS by the LTE system have been reported [6]-[9]. Also, some simulation based analysis of LTE system and its coexistence in the TVWS have been performed [9]-[11]. However, for further investigation of the LTE system in the TVWS regarding effectiveness of traffic offloading, evaluation based on an actual TVWS LTE system that consists of an LTE base station (eNB, evolutional Node B) and terminal adaptors (UE, User Equipment) is strongly required. In this study, we prototyped TDD/FDD (Time/Frequency Division Duplex) dual mode LTE eNB and UE enabling TVWS communication by applying the UHF (Ultra High Frequency) converter technology [12][13] and RF performance was characterized.

The rest of this paper is organized as follows. In Section II, our developing LTE system in TVWS is introduced and hardware prototypes including architecture and design are described. In Section III, measured RF performance and spurious response are described. Finally, we conclude this paper in Section IV.

\section{HARDWARE PROTOTYPES}

\section{A. LTE System in TVWS}

A TVWS LTE system under development in NICT consists of a TVWS database, a network manager, a coexistence manager, a TVWS eNB and UEs, as shown in Fig. 1. The TVWS database developed by NICT [14], which has information about primary users (e.g. location, antenna height, transmitting power and surrounding terrain) provides an available channel list to the TVWS eNB, when receiving an inquiry via the Internet. The network manager and the coexistence manager provide interference information between 


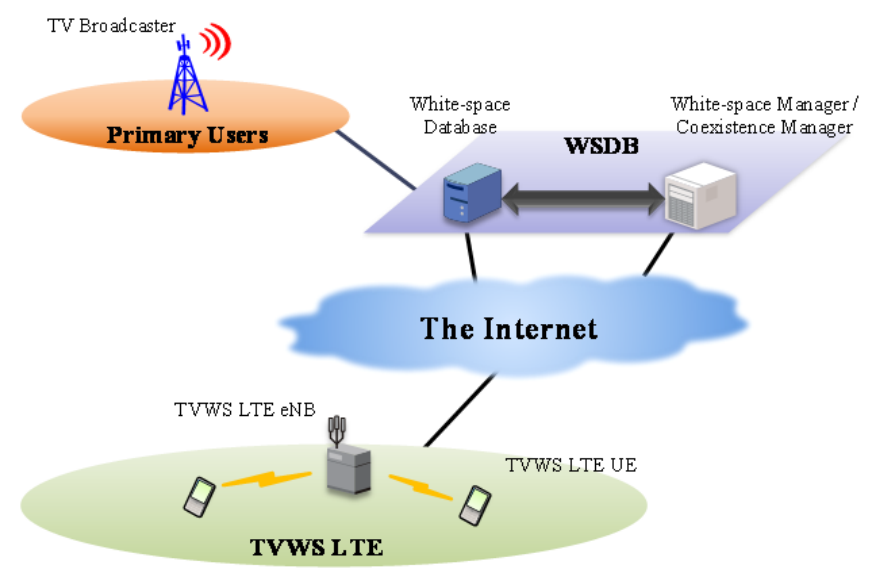

Figure 1. TVWS LTE system.

secondary systems and allocate TVWS channels to each system. Therefore, radio parameters of TVWS eNB and UE (e.g. a communication frequency, a duplex mode and output power) are required to be dynamically changed under the control of the TVWS database and the network manager.

Fig. 2 shows prototyped TVWS eNB and UE that are available in the TVWS. To realize communication in the TV band, UHF converters are connected to off-the-shelf LTE eNB and data card, and convert a communication frequency from the existing band to the UHF band. In this study, the LTE system is based on Release 8 and a $2.6 \mathrm{GHz}$ band is used as the existing band: i.e. Band $38(2,570-2,620 \mathrm{MHz})$ for the TDD mode and Band 7 (Uplink : 2,500-2,570 MHz, Downlink : 2,620-2,690 MHz) for the FDD mode. A covered frequency range in the TV band is $470-710 \mathrm{MHz}$.

\section{B. Prototyped TVWS eNB}

Fig. 3 shows hardware architecture of the prototyped TVWS eNB. The off-the-shelf eNB consists of three main boards. One is a baseband board based on the LTE Release 8 and the other two are RF boards for FDD Band 7 and TDD Band 38. A UHF converter with its control board is connected to both TDD and FDD RF boards separately and converts an RF frequency from $2.6 \mathrm{GHz}$ to UHF. Since an interface of the control board is the USB (Universal Serial Bus) interface, an

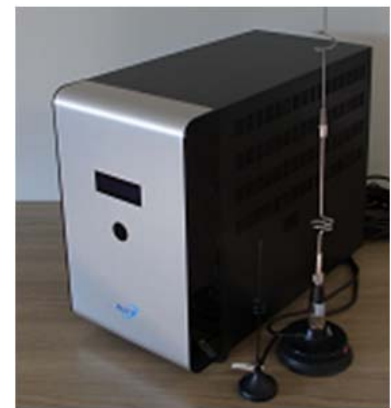

(a) Base station (eNB)

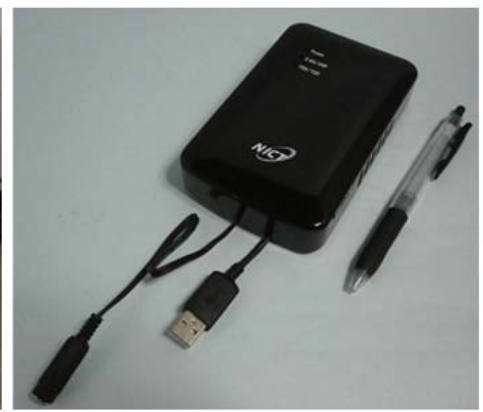

(b) USB terminal adapter (UE)
Figure 2. TVWS LTE hardware prototype. additional CPU board is implemented between the control board and a VPN (Virtual Private Network) router to convert a control signal from the Ethernet to the USB signal. By this means, radio parameters of the TVWS eNB can be changed dynamically under the control of the white-space database and the network manager via the Internet. Fig. 4 shows the prototyped UHF converter and the control board assembled in the TVWS eNB. Single-sided mounting boards are employed to reduce total thickness and both boards are connected inplane to each other with an on-board connector. To meet a tight requirement of frequency accuracy in the LTE communication system, an oven controlled crystal oscillator (OCXO) is implemented in the TVWS eNB and provides a precise 10 $\mathrm{MHz}$ reference clock to the baseband board, two RF boards and the UHF converter.

Target specifications of the TVWS eNB is summarized in Table I. Peak output power is designed to $1 \mathrm{~W}$ according to the FCC rule for the Fixed TV band devices [3]. Also, by implementing an output power adjustment function by more than $20 \mathrm{~dB}$, the TVWS eNB can be operated under a variety of rules regulated by different countries or regions. Furthermore, this adjustment function can flatten frequency characteristics of output power in a wide frequency range of the TV band.

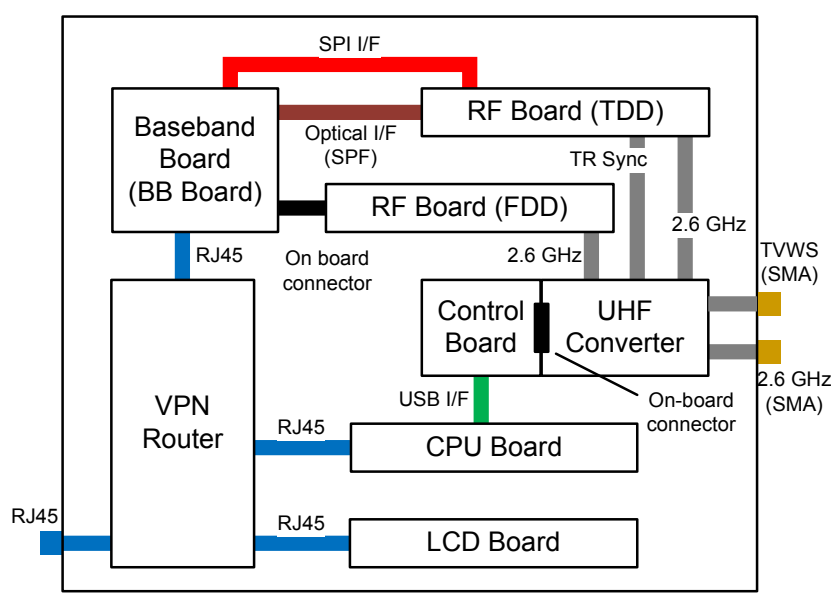

Figure 3. Hardware architecture of the prototyped TDD/FDD dual mode eNB.

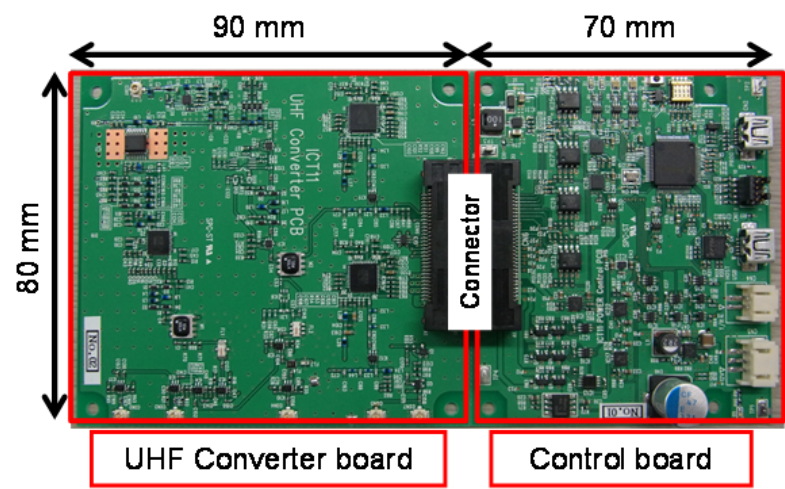

Figure 4. Prototyped UHF converter and control board in the TVWS LTE eNB. 
TABLE I. TARGET SPECIFICATIONS OF PROTOTYPED HARDWARES.

\begin{tabular}{lcc}
\hline \hline Item & TVWS LTE eNB & TVWS LTE UE \\
\hline Communication system & \multicolumn{2}{c}{ Based on LTE Release 8} \\
Frequency range & \multicolumn{2}{c}{$470-710 \mathrm{MHz}$} \\
Carrier frequency error & $< \pm 0.05 \mathrm{ppm}$ & $< \pm 0.1 \mathrm{ppm}$ \\
Duplex system & FDD $/$ TDD \\
Bandwidth & \multicolumn{2}{c}{$5,10,20 \mathrm{MHz}$} \\
Duplex spacing (FDD) & $>80 \mathrm{MHz}$ & $>80 \mathrm{MHz}$ \\
Output power (peak) & $\mathrm{Up} \mathrm{to}+30 \mathrm{dBm}$ & $\mathrm{Up} \mathrm{to}+20 \mathrm{dBm}$ \\
Power tuning range & $>20 \mathrm{~dB}$ & $>20 \mathrm{~dB}$ \\
EVM & $<12.5 \%$ & $<12.5 \%$ \\
Receiver sensitivity & $<-90 \mathrm{dBm}$ & $<-90 \mathrm{dBm}$ \\
(QPSK, throughput $95 \%)$ & -15 to $-90 \mathrm{dBm}$ & -15 to $-90 \mathrm{dBm}$ \\
Receiver dynamic range & $170(\mathrm{~W}) \times 420(\mathrm{~L}) \times$ & $84 \times 133 \times 30 \mathrm{~mm}^{3}$ \\
Size & $300(\mathrm{H}) \mathrm{mm}^{3}$ & \\
\hline \hline
\end{tabular}

Receiving sensitivity of the TVWS eNB is designed to -90 $\mathrm{dBm}$. This receiving sensitivity is about $10 \mathrm{~dB}$ higher than $-100.8 \mathrm{dBm}$ in the LTE standard due to deterioration of total noise figure (NF) by connecting the UHF converter and interference of transmission signal and noise to a receiver part especially in the FDD mode.

\section{Prototyped TVWS UE}

Fig. 5 shows hardware architecture of the prototyped TVWS UE. A UHF converter is connected to the off-the-shelf LTE data card supporting both TDD/FDD modes and converts its RF frequency from the $2.6 \mathrm{GHz}$ band to the UHF band. Both the off-the-shelf LTE data card and the control board for the UHF converter have a USB interface and a USB hub is implemented to bundle into one USB port. Therefore, radio parameters of the LTE data card and the UHF converter can be dynamically controlled by external equipment such as a personal computer, independently. Fig. 6 shows the UHF converter and the control board in the TVWS UE. Doublesided mounting boards are employed and both boards are stacked via a connector to reduce occupied area. To alleviate a frequency drift of the reference clock mounted on the UHF converter board at high temperature, passive heat sinks are attached to power amplifiers and PLL ICs, and heat radiation slits are also formed on side faces of the case.

Peak output power of the TVWS UE is designed to 100 $\mathrm{mW}$ according to the FCC rule for the Mode II TV band devices (personal or portable device with a database access capability) [3]. A power adjustment function by more than 20 $\mathrm{dB}$ is also implemented, as shown in Table I. This power adjustment function is implemented in the UHF converter and output power of the off-the-shelf LTE data card is limited to preferable level by using a Pmax parameter. Receiving sensitivity of the TVWS UE is designed to $-90 \mathrm{dBm}$, as well as the prototyped TVWS eNB, by considering NF deterioration and interference of transmission signal and noise to a receiver part.

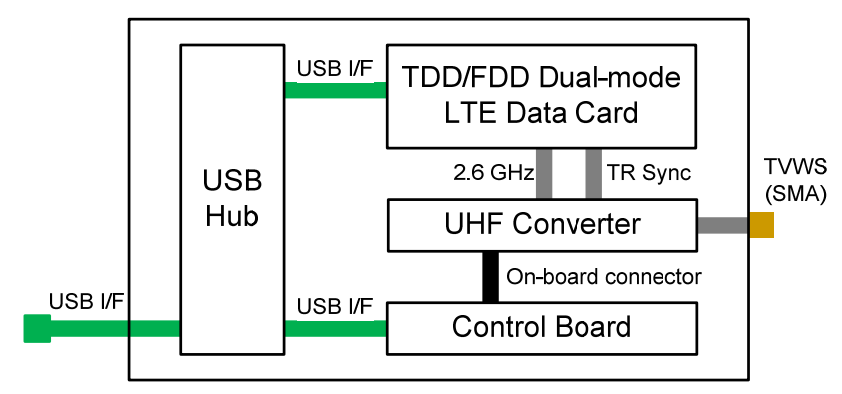

Figure 5. Hardware architecture of the prototyped TDD/FDD dual mode UE.

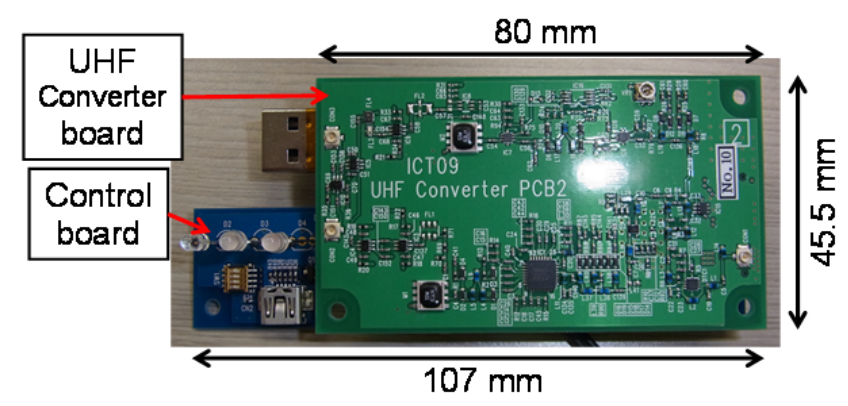

Figure 6. Prototyped UHF converter and control board in the TVWS LTE UE.

\section{UHF Converter for TDD/FDD Dual Mode LTE System}

Fig. 7 and 8 show block diagrams and level charts in a transmitter and a receiver of the UHF converter for the prototyped TVWS UE, respectively. Here, levels in both level charts indicate mean power. In the prototyped TVWS eNB, almost same architecture is employed except for the power amplifier. A power amplifier in this TVWS eNB requires higher linearity to enable an output power of $1 \mathrm{~W}$ for the OFDM (Orthogonal Frequency-Division Multiplexing) system.

For the FDD mode in the TVWS, pair band frequencies for a transmission and a reception are preferred to be determined independently, unlike the existing LTE system. Furthermore, narrower duplex spacing between pair bands contributes to more available combinations of pair bands and enhances efficiency of spectrum resource utilization. Therefore, a frequency variable duplexer, which can set arbitrary pair bands with narrow duplex spacing, is required to avoid interference of transmission signal and noise to a receiver in the FDD mode. In this prototype, a frequency variable duplexer which consists of a wide band coupler and variable band rejection filters (VBRFs) is designed and implemented for supporting the FDD mode. Here, an uplink frequency is set to a lower frequency band of the pair bands, and thus, the VBRF implemented in the receiver part for the TVWS UE rejects the lower frequency band of the transmission. In reverse, the VBRF for the TVWS eNB rejects a higher frequency band.

The transmitting part has a variable gain driver amplifier in the UHF part after passing through a mixer and output power in the UHF band is adjusted to $+15 \mathrm{dBm}$ at an input signal level of $+18 \mathrm{dBm}$ in the $2.6 \mathrm{GHz}$ band. Consequently, peak power 

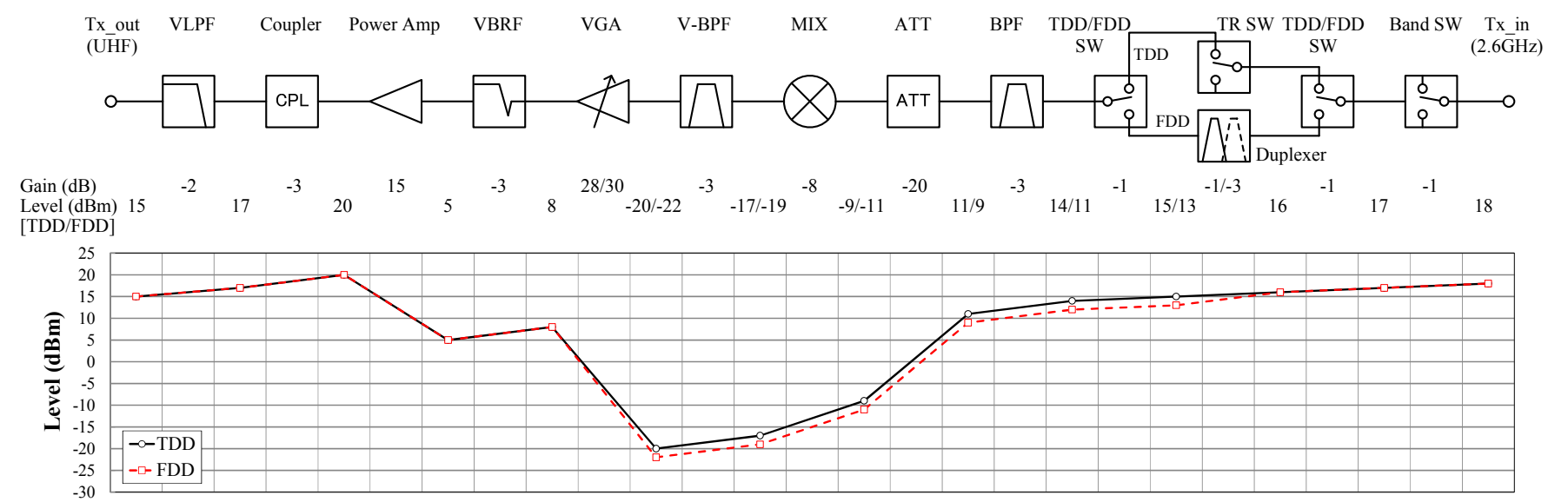

Figure 7. Block diagram and level chart in a transmitter of the UHF converter for the TVWS LTE UE.
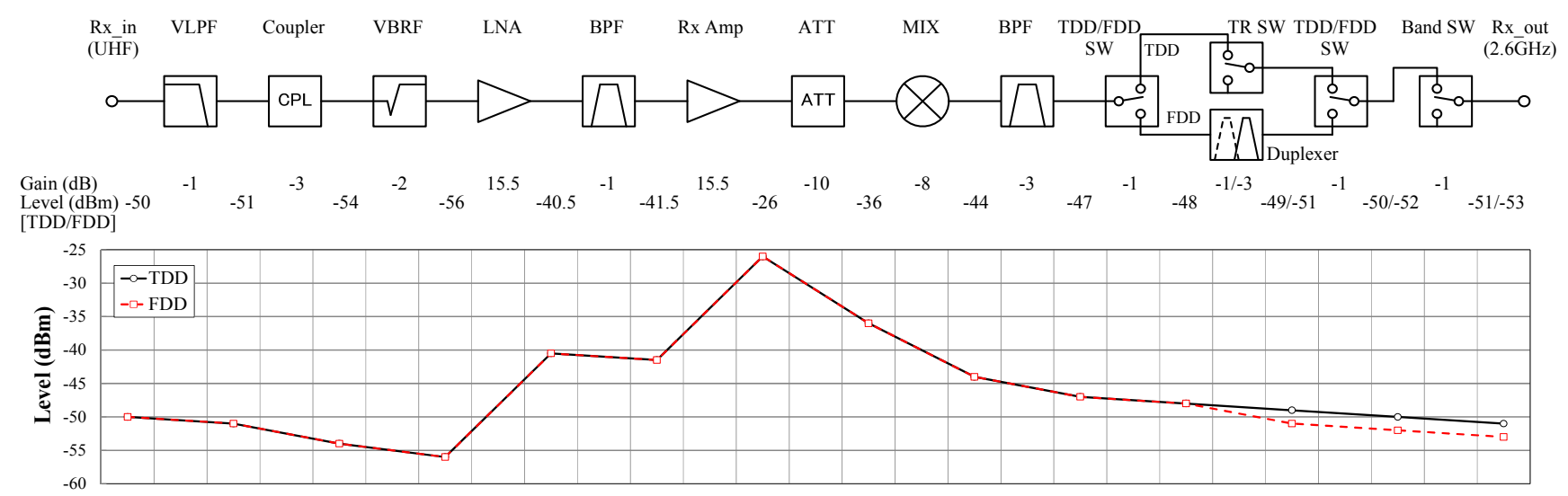

Figure 8. Block diagram and level chart in a receiver of the UHF converter for the TVWS LTE UE.

in the UHF band does not exceed $+20 \mathrm{dBm}$. In the FDD mode, the off-the-shelf duplexer for Band 7 is inserted in a $2.6 \mathrm{GHz}$ interface, and thus, total loss in the FDD mode is $2 \mathrm{~dB}$ higher than that in the TDD mode. To compensate such higher loss, gain of the driver amplifier in the FDD mode is set to $2 \mathrm{~dB}$ higher than that in the TDD mode.

In the receiver part, total gain is set to $-1 \mathrm{~dB}$ for the TDD mode. In general, it is preferred to set higher gain to the UHF converter and minimize deterioration of total NF, to obtain better receiving sensitivity. However, if high gain is set to the UHF converter, received signal level in the $2.6 \mathrm{GHz}$ signal to the off-the-shelf LTE data card become higher than actual signal level in the UHF band, and the RSSI (Received Signal Strength Indicator) level in the data card increases according to its gain. As a result, output power in the UHF band is limited to lower level than required and a connection failure problem might occur. Therefore, total gain of the UHF converter is designed to about $0 \mathrm{~dB}$ in this prototype. In addition, total loss in the FDD mode is also $2 \mathrm{~dB}$ higher than that in the TDD mode in the receiver part. Therefore, receiving sensitivity in the FDD mode is simply $2 \mathrm{~dB}$ deteriorated in comparison to that in the TDD mode.

\section{Performance Evaluation of Prototyped UE}

\section{A. RF Characteristics}

Regarding the off-the-shelf LTE data card implemented in the TVWS UE, technical specifications such as a test mode are not disclosed at this stage. Therefore, RF characteristics of the TVWS UE have been evaluated by using a radio communication tester (MT8820, Anritsu), in this study. The prototyped TVWS eNB uses the same architecture as the TVWS UE, so that only the RF characteristics of the TVWS $\mathrm{UE}$ are described in this section.

Table II shows measured RF characteristics of the TVWS UE. RF characteristics in the TVWS for both TDD and FDD modes are compared with those in the existing bands, i.e. Band 38 and Band 7, respectively. As shown in Table II, $2.595 \mathrm{GHz}$ in Band 38, and $2.535 \mathrm{GHz}$ for an uplink and $2.655 \mathrm{GHz}$ for a downlink in Band 7 are used as frequencies in the existing bands and converted to the UHF band by mixing with an upper local. Signal bandwidth (BW) is $5 \mathrm{MHz}$ and a duplex spacing for the FDD mode in the TVWS is set to about $90 \mathrm{MHz}$.

Transmitting performance for both TDD and FDD modes are measured on the conditions of the level diagram in Fig. 7. 
TABLE II. MEASURED RF CHARACTERISTICS IN TVWS COMPARED WITH NATIVE CHARACTERISTICS.

\begin{tabular}{|c|c|c|c|c|c|c|c|c|c|}
\hline \multirow[b]{2}{*}{ Item } & \multirow[b]{2}{*}{ Unit } & \multicolumn{4}{|c|}{ TDD } & \multicolumn{4}{|c|}{ FDD } \\
\hline & & Native & $\begin{array}{l}\text { Lower } \\
\mathrm{CH}\end{array}$ & $\begin{array}{c}\text { Middle } \\
\text { CH }\end{array}$ & $\begin{array}{l}\text { Upper } \\
\text { CH }\end{array}$ & Native & $\begin{array}{c}\text { Lower } \\
\mathrm{CH}\end{array}$ & $\begin{array}{c}\text { Middle } \\
\mathrm{CH}\end{array}$ & $\begin{array}{c}\text { Upper } \\
\mathrm{CH}\end{array}$ \\
\hline Tx carrier frequency & $\mathrm{MHz}$ & 2595 & 473 & 572 & 767 & 2535 & 473 & 572 & 668 \\
\hline Carrier frequency error & ppm & 0.00 & 0.03 & 0.02 & 0.01 & 0.00 & -0.03 & 0.00 & -0.01 \\
\hline Rx carrier frequency & $\mathrm{MHz}$ & \multicolumn{4}{|c|}{ N/A } & 2655 & 572 & 671 & 767 \\
\hline Bandwidth & $\mathrm{MHz}$ & \multicolumn{4}{|c|}{5} & \multicolumn{4}{|c|}{5} \\
\hline Tx power (average) & $\mathrm{dBm}$ & 18.37 & 13.53 & 14.31 & 15.29 & 17.93 & 13.71 & 13.72 & 14.19 \\
\hline EVM & $\%$ & 3.52 & 3.87 & 6.72 & 4.35 & 4.39 & 5.75 & 8.11 & 5.18 \\
\hline OBW & $\mathrm{MHz}$ & 4.455 & 4.455 & 4.477 & 4.455 & 4.455 & 4.455 & 4.477 & 4.455 \\
\hline Receiver sensitivity & $\mathrm{dBm}$ & -96 & -95 & -93 & -94 & -97 & -89 & -94 & -85 \\
\hline
\end{tabular}

Carrier frequency deviation in the TV band is sufficiently smaller than $\pm 0.1 \mathrm{ppm}$ in specifications of the existing LTE system. Measured output power almost agrees with a target output power of $+15 \mathrm{dBm}$. In this result, output power tends to be higher in a high frequency range of the TV band, however, a fluctuation of about $1.5 \mathrm{~dB}$ can be flattened by setting appropriate gain of the driver amplifier on the UHF converter for each channel, individually. Regarding modulation accuracy, measured EVM (Error Vector Magnitude) in the TVWS is slightly deteriorated compared with that in the existing LTE band, however, it is sufficiently lower than $12.5 \%$ in specifications of the LTE system. Meanwhile, EVM in 572 $\mathrm{MHz}$ is worse than that in other frequencies for both TDD and FDD modes. This deterioration is caused by spurious that is generated close to the carrier frequency by the mixer on the UHF converter, and thus, the occupied bandwidth (OBW) is also slightly widened. It was confirmed that this spurious was derived from a half local generated by a PLL and VCO IC and can be suppressed by inserting a high pass filter in a local signal line.

Receiving sensitivity was measured as an input signal level on the condition of $95 \%$ throughput with a QPSK (Quadrature Phase Shift Keying) modulation according to specifications in the standard of the existing LTE system. In the TDD mode, receiving sensitivity is about -93 to $-95 \mathrm{dBm}$ and satisfies a designed receiving sensitivity of $-90 \mathrm{dBm}$. A deterioration of $1-3 \mathrm{~dB}$ in comparison to that in the existing band as shown in Table II is mainly caused by NF deterioration due to a $0 \mathrm{~dB}$ gain of the UHF converter. Measured NF of the UHF converter is about $8-10 \mathrm{~dB}$ and might be higher than that of the off-theshelf LTE data card. In the FDD mode, remarkable deterioration of receiving sensitivity was observed at higher and lower channels in the TV band. This is mainly caused by insufficient frequency characteristics of the wide band coupler in the frequency variable duplexer, and interference of the transmission signal and noise to the receiver part is not sufficiently suppressed at those channels. Since the frequency range in the TV band is very wide of $470-710 \mathrm{MHz}$ in Japan, $\mathrm{RF}$ circuit and component with a wider BW is one of main challenges to develop TV band portable devices. In this prototype, the implemented wide band coupler is composed of discrete components and does not sufficiently cover a whole
TV band at this stage and fine adjustment of circuit parameters is necessary to each channel, individually. For further improvement, new technologies such as a tunable device supporting a wide frequency range of the TV band are expected to solve this problem.

\section{B. Spurious Response}

Spurious response of the prototyped UHF converter is measured with using a signal generator (SMU200A, Rohde\& Shwartz) and a signal analyzer (FSQ8, Rohde\&Shwartz). Fig. 9 shows typical spurious response in the TV band and a wider frequency range up to $3 \mathrm{GHz}$. A carrier frequency of $671 \mathrm{MHz}$ is generated by mixing an LTE signal of $2,595 \mathrm{MHz}$ and an upper local of 3,266 MHz. In this measurement, a single carrier with a $5 \mathrm{MHz} \mathrm{BW}$ and QPSK modulation for the up-link is used and mean output power in the TV band is set to about +15 $\mathrm{dBm}$. In addition, spurious response is measured by using a max hold with a resolution bandwidth (BRW) of $100 \mathrm{kHz}$.

In TV band, no spurious that exceeds $-52.8 \mathrm{dBm} / 100 \mathrm{kHz}$ indicated in the FCC rule [3] is observed as shown in Fig. 9(a). On the other hand, 2nd harmonics of the carrier frequency is observed in Fig. 9(b). This spurious level varies by the combination of carrier and local frequencies and higher spurious level is observed in some conditions. Spurious at higher frequency including 2nd harmonics is supposed to be suppressed by a variable low pass filter (VLPF) implemented just after an antenna port and it is confirmed that fine adjustment of VLPF circuit parameters can suppress such spurious for further improvement.

\section{CONCLUSION}

In this study, hardware design and prototype of TDD/FDD dual mode TVWS eNB and UE are described and RF performance is characterized. In both prototypes, the UHF converter is implemented to convert a communication frequency from the existing $2.6 \mathrm{GHz}$ band to the UHF band. RF performance and spurious response of the LTE UE are also measured and almost good performance was obtained except for deterioration of receiving sensitivity at higher and lower channels in the FDD mode. This deterioration is mainly caused by frequency characteristics of the wide band coupler implemented in the frequency variable duplexer on the UHF 


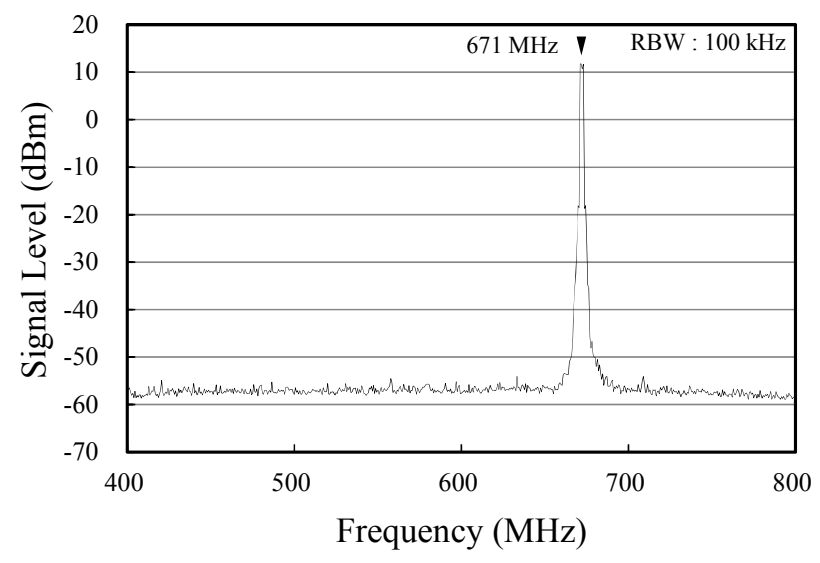

(a) In the TV band.

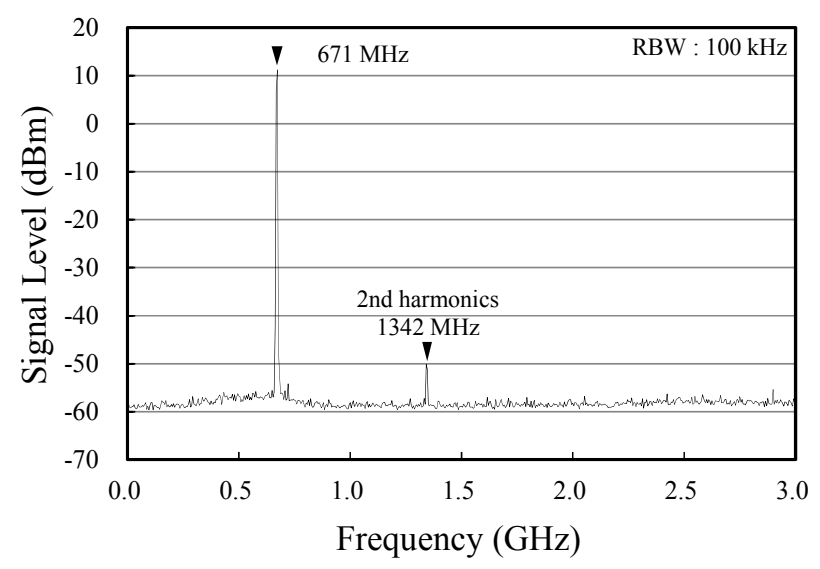

(b) In a wide frequency range.

Figure 9. Spurious response. converter and will be improved by fine adjustment of circuit parameters for each channel. As a future work, connectivity between TVWS eNB and UE are evaluated for further evaluation of effectiveness of traffic offloading.

\section{REFERENCES}

[1] IEEE802.11af Task Group. http://www.ieee802.org/11/Reports/tgaf update.htm

[2] IEEE802.22 Working Group. http://www.ieee802.org/22/.

[3] FCC, "Unlisenced Operation in the TV Broadcast Bands, Third Memorandum Opinion and Order," FCC. 12-36, Apr. 5, 2012.

[4] Ofcom, "Regulatory Requirements White Space Devices in the UHF TV band," July 2012.

[5] H. Harada, "Status report on usage of TV white space in Japan," 802.1112/677r0, May 2012.

[6] H. Harada, K. Ishidu, H. Murakami, K. Ibuka and T. Matsumura, "Research and development on white space LTE system," IEICE Technical Report, vol. 112, no. 351, RCS2012-218, pp. 209-213, Dec. 2012 (in Japanese).

[7] M. Rahman and A. Behravan, "License-exempt LTE systems for secondary spectrum usage: Scenarios and first assessment," in Proc. IEEE DYSPAN2011, pp. 349-358, May 2011.

[8] M. Fitch, M. Nekovee and S. Kawade, "Wireless service provision in TV white space with cognitive radio technology: A telecom operator's perspective and experience," IEEE Communications Magazine, pp. 64$73,2011$.

[9] Z. Zhao and M. Schellmann, "Interference study for cognitive LTEFemtocell in TV white spaces," in Proc. ITU WT 2011, pp. 153-158, Oct. 2011.

[10] J. Xiao, F. Ye, T. Tian and R. Q. Hu, "CR Enabled TD-LTE within TV White Space: System Level Performance Analysis," in Proc IEEE GLOBECOM2011, Dec. 2011.

[11] M. Beluri, et al., "Mechanisms for LTE coexistence in TV white space," in Proc. IEEE DySPAN2012, pp. 317-326, Oct. 2011.

[12] T. Matsumura and H. Harada, "Prototype of UHF converter for TV white-spaces utilization," in Proc. WPMC2012, pp. 123-127, Sep. 2012.

[13] T. Matsumura and H. Harada, "Prototype of Tablet-type TV Band Portable Device with UHF Converter for TV White-spaces Utilization," in Proc. PIMRC2013, pp. 2748-2752, Sep. 2013.

[14] K. Ishizu, H. Murakami and H. Harada, "TV white space database for coexistence of primary-secondary and secondary-secondary systems in mexh networking," in Proc. WPMC2012, pp. 118-122, Sep. 2012. 